\title{
Benchmarking und Qualitätssicherung in der Senologie
}

\section{H. G. Bender}

Das Bemühen um Behandlungsqualität ist ohne Frage ein zentrales Anliegen jeder ärztlichen Behandlung. Treibende Kraft war dabei traditionell der karitative Aspekt des ärztlichen Handelns, das erst in den letzten Jahrzehnten durch ökonomische und „Marketing“-Gesichtspunkte ergänzt und zum Teil ersetzt wurde. Qualität der Therapie wird nicht nur der individuellen Patientin geschuldet, sondern wird von Kostenträgern eingefordert und von politischen Planern als Tätigkeitsvoraussetzung verlangt.

Für das Mammakarzinom hat sich bezüglich der Entwicklung von strukturierten Qualitätskonzepten eine Vorreiterfunktion ergeben. Diese Tatsache erklärt sich dadurch, dass durch die Erkrankungshäufigkeit und evidente Qualitätsmängel durch persönliches Fehlverhalten eine politische Aktion mit dem Ziel einer besser kontrollierbaren Qualität und der Etablierung von Brustzentren ausgelöst wurde. Darüber hinaus hat die im Vergleich mit anderen onkologischen Erkrankungen intensive Entwicklung in Richtung multimodaler Behandlungsstrategien eine überschaubare Dokumentation nahegelegt.

Beim Start der Bemühungen wurde deutlich, dass Qualitätssicherung und darauf basierende Vergleiche aus unterschiedlichen Interessen betrieben und für diese konfiguriert und zum Teil auch missbraucht werden können. Dies wurde deutlich, als im Jahr 2002 ein intensiv erarbeitetes Dokumentationskonzept zur Begleitung des DMP-Programms für das Mammakarzinom (WBC) durch eine Grobdokumentation als Zugang zum Risikostrukturausgleich ersetzt wurde, die keinen Zusammenhang mit Qualitätsaspekten hatte. In der Folge wurden Dokumentationsprogramme aus verschiedenen Richtungen und Intentionen in die Praxis eingeführt, wodurch unrealistische Mehrfachdokumentationsanforderungen - zumeist an ärztliche Mitarbeiter resultierten.

Von ärztlicher Seite ist von Beginn an darauf hingewiesen worden, dass eine Entwicklung und Verteilung der Brustzentren auf der Basis von Qualitätsmerkmalen die einzig allgemein akzeptable Vorgehensweise darstellt. Unter diesem Gesichtspunkt muß dann auch die Qualitätserfassung und das darauf basierende
Benchmarking, das für einzelne Ärzte und Abteilungen existentielle Bedeutung bekommen kann, einen allgemein akzeptierten, medizinisch definierten Standard erfüllen, der nicht wegzudiskutieren ist.

Dabei muß der Aufwand für die Erfüllung dieses Zieles limitiert werden - nicht zuletzt, weil die Kosten und der ärztliche und sonstige Zeitaufwand in unmittelbarer Konkurrenz zu der eigentlichen Patientenversorgung stehen: Wenn der letzte Euro für die perfekte Dokumentation verausgabt wurde, stehen für Operation, Bestrahlung und medikamentöse Therapie keine Ressourcen mehr zur Verfügung.

Des Weiteren sollte so früh wie möglich die Frage geklärt werden, welche Qualitätsunterschiede in einzelnen Subgruppen auf der Grundlage der gegebenen Fallzahlen statistisch valide herausgearbeitet werden können und welche theoretisch darstellbaren Differenzen für die praktische Betreuung der individuellen Patientin relevant sind. Statt grenzwertiger p-Werte sollten möglicherweise Qualitätskorridore dargestellt werden, die guten Standard, verbesserungsfähige Ergebnisse und nicht vertretbare Verhältnisse erkennbar machen.

Das - verständliche - Streben von Patientinnen nach der optimalen Therapiequalität sollte nicht in Frustration und in Vorwürfen gegen die Ärzteschaft enden, dass die erhoffte Darstellung der Qualität von Anbeginn auf Grund der Ausgangsbedingungen gar nicht erreichbar war und diese Tatsache bei systematischer Analyse der Situation von Anfang an hätte erkannt werden können.

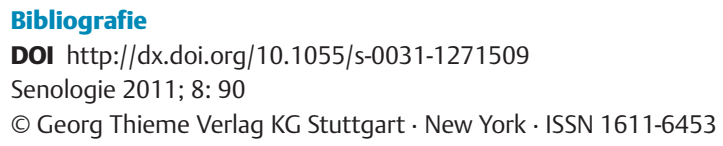

benderhg@med.uni-duesseldorf.de 